\title{
Management of Fetal Supraventricular Tachycardia: Case Series from a Tertiary Perinatal Cardiac Center
}

\author{
Elisha Broom $^{a}$ Joseph T. Thomas ${ }^{a, b}$ Scott Petersen ${ }^{a, b} \quad$ Alex Gooi $^{c}$ \\ Cameron Ward $^{c}$ Glenn Gardener ${ }^{\mathrm{a}, \mathrm{b}}$ Karam Kostner ${ }^{\mathrm{d}}$ Alison Lee-Tannock ${ }^{\mathrm{a}}$ \\ Sailesh Kumar a, b, e \\ ${ }^{a}$ Mater Centre for Maternal Fetal Medicine, Mater Mother's Hospital, South Brisbane, QLD, Australia; ${ }^{b}$ Mater \\ Research Institute-University of Queensland, Aubigny Place, Raymond Terrace, South Brisbane, QLD, Australia; ${ }^{\mathrm{C}} \mathrm{Qld}$ \\ Paediatric Cardiac Service, Queensland Children's Hospital, South Brisbane, QLD, Australia; 'Department of Adult \\ Cardiology, Mater Health Services, South Brisbane, QLD, Australia; 'Department of Obstetrics and Gynaecology, \\ Faculty of Medicine, The University of Queensland, Herston, QLD, Australia
}

\section{Keywords}

Arrhythmia · Supraventricular tachycardia · Fetal tachycardia · Echocardiography

\begin{abstract}
Background: Fetal supraventricular tachycardia is a relatively uncommon cardiac rhythm abnormality which is often associated with adverse perinatal outcomes if untreated. Although there are several treatment modalities and protocols in use globally, there is no consensus as to the most effective antiarrhythmic to manage this condition. Aim: This study aimed to evaluate perinatal outcomes following prenatal maternal therapy for fetal supraventricular tachycardia. $\mathbf{M a -}$ terials and Methods: This was a 20-year retrospective cohort study. Institutional records were reviewed for antenatal therapy choice and maternal and fetal outcomes. Results: Sixtynine cases met diagnostic criteria for fetal SVT, of which 56 $(81 \%)$ received maternal antiarrhythmic therapy. Digoxin was the most common, but least effective, first-line therapy in 28 patients, achieving successful rate reversion in $35.7 \%$. Thirty-one patients (55\%) required second-line therapy, and this was most successful with digoxin and flecainide poly-
\end{abstract}

karger@karger.com www.karger.com/fdt

Karger"

BOPEN ACCESS
(C) 2021 The Author(s)

Published by S. Karger AG, Basel

This is an Open Access article licensed under the Creative Common Attribution-NonCommercial-4.0 International License (CC BY-NC) (http://www.karger.com/Services/OpenAccessLicense), applicable to the online version of the article only. Usage and distribution for commercial purposes requires written permission. therapy achieving rate reversion in 17 of 18 cases (94.5\%) at a median of 3 days (1.5-7). Hydrops was present in $23(33 \%)$ cases at initial presentation, 16 of which achieved rate reversion. There was minimal difference in treatment efficacy comparing single- or multiple-agent treatment in the setting of hydrops ( $50 \%$ vs. $42.8 \%)$. Side effects occurred in $14 / 56$ treated patients $(25 \%)$ but were severe in only $8(14.3 \%)$ women, most commonly with digoxin and flecainide polytherapy ( 6 of 8 cases). There were 3 (4\%) fetal deaths amongst the study cohort. Conclusions: Digoxin and flecainide polytherapy were well tolerated and successfully achieved rhythm and rate control in fetuses with prenatally diagnosed supraventricular tachycardia. The presence of hydrops was a poor prognostic feature.

(c) 2021 The Author(s)

Published by S. Karger AG, Basel

\section{Introduction}

Fetal supraventricular tachycardia (SVT) is a cardiac rhythm anomaly complicating approximately $1: 1,000$ pregnancies [1]. Although in the majority of cases this is an isolated abnormality, a concomitant structural heart
Correspondence to:

Elisha Broom, elisha.broom@mater.org.au 
defect is present in $5-10 \%$ of cases [2]. Fetal SVT accounts for up to $90 \%$ of all fetal tachyarrhythmias [3] and in most instances arises from retrograde atrial activation across a fast conducting accessory pathway $[2,4]$. Postnatally, up to $10 \%$ of infants will have Wolff-Parkinson-White syndrome, the majority of which will resolve in the first year of life [2].

Sustained fetal SVT can lead to complications related to poor cardiac output. These include hydrops fetalis in $20-30 \%$ of cases - if untreated fetal demise and/or maternal mirror syndrome can ensue $[5,6]$. Neonatal outcomes are improved if reversion to sinus rhythm and resolution of hydrops is achieved prior to birth [7].

Management of these rare cases is often difficult and requires prolonged maternal antiarrhythmic treatment to achieve adequate transplacental transfer and therapeutic drug levels in the fetus. As there is no clear consensus regarding the choice of antiarrhythmic agent, duration of treatment, or timing of birth if maternal treatment is unsuccessful in achieving cardioversion, clinical management is often individualized. The aim of this study thus was to review outcomes of all cases of fetal SVT managed at a single Australian tertiary perinatal cardiac center.

\section{Methods}

\section{Patient Selection}

We conducted a retrospective observational study of all cases of fetal SVT treated at the Queensland Paediatric Cardiac Services and Maternal Fetal Medicine (MFM) department at the Mater Mother's Hospital in Brisbane Australia between 2000 and 2020. Maternal demographic data (parity, gestation), ultrasound findings (presence or absence of hydrops, type of SVT and overall fetal heart rate, umbilical artery, middle cerebral artery, and ductus venosus Doppler), and clinical outcomes (rate reversion, maternal side effects, delivery timing and mode, birth weight, selected fetal outcomes) were extracted from the institution's electronic health records and ultrasound reporting system (Viewpoint ${ }^{\circledR} \mathrm{GE}$ ). Where necessary, this was supplemented by individual chart review to obtain details of duration and gestation at commencement of treatment, time to achieve sinus rhythm, details of dose adjustment, and need for polytherapy. Ethics and Governance approvals (HREC/14/MHS/37) were granted by the Mater Human Research Ethics Committee and Governance Office, respectively.

All cases were assessed by a fetal cardiologist and an MFM specialist. In addition to a detailed structural survey, fetal echocardiography was also performed. Fetal tachycardia was defined as a fetal heart rate of above $180 \mathrm{bpm}$ and SVT defined as 1:1 AV conduction based on M-mode echocardiography. SVT was occasionally further characterized based upon the ventriculo-atrial (VA) interval [8]. Atrial flutter was diagnosed when the fetal heart rate was $>250 \mathrm{bpm}$ with 2:1 AV conduction. Fetal hydrops was defined as the presence of fluid in two or more fetal compartments.
If the diagnosis of fetal SVT was confirmed, women were counseled about management options and maternal antiarrhythmic therapy then commenced. Pretreatment maternal renal and liver function was checked and electrocardiogram and echocardiography performed in all cases. Antiarrhythmic agents used included digoxin, flecainide, and sotalol either as single-agent therapy or in combination. All women were admitted for in-patient monitoring when antiarrhythmic was commenced with subsequent outpatient management once the fetal heart rate had normalized. Maternal side effects were recorded as mild (nausea, dizziness, and fatigue) or severe (abnormal liver function tests, chest pain, abnormal electrocardiogram, or side effects severe enough to precipitate delivery). After commencement of maternal therapy, the fetal condition was closely monitored with regular ultrasound scans $1-2$ times a week until delivery. Management of cases varied slightly according to the individual treating pediatric cardiologist.

\section{Results}

Over the study period, there were 69 cases that met the diagnostic criteria for fetal SVT. Of these, $56(81 \%)$ received maternal antiarrhythmic therapy (Table 1). The median gestational age at diagnosis was $29+2$ weeks $(26+0-33+4)$ and $36+1$ weeks $(34+0-38+4)$ at birth. Hydrops was present in $23(33.3 \%)$ cases. Only one fetus had a structurally abnormal heart with multiple rhabdomyomas. Most cases of SVT were unclassified as to subtype $(51 / 69)(73.9 \%)$, with only 7 cases $(10 \%)$ of atrial flutter within the cohort.

Overall, first-line therapy was unsuccessful in 31 of 56 (55.4\%) cases (Fig. 1). Of these, 4 women delivered because of advanced gestation and/or maternal side effects and 27 women went on to second-line therapy with different agents following a median first-line treatment duration of 5 days (3-9). Overall success rates of secondline therapy in these 27 patients were high with 23 cases (85\%) ultimately achieving rate reversion. The most successful second-line therapy was digoxin and flecainide polytherapy which achieved rate reversion in 17 of 18 cases $(94.5 \%)$ following a median treatment duration of 3 days (1.5-7).

Digoxin was the most common first-line therapy and used in 28 women (50\%). Flecainide was utilized as firstline therapy in $7(12.5 \%)$ and sotalol in $6(10.7 \%)$ women, respectively (Table 2 ). Successful conversion to sinus rhythm occurred in 10 cases (35.7\%) in the digoxin group following a median treatment of 4.5 days (3-9) of maternal treatment. However, when compared to digoxin, both flecainide and sotalol achieved higher rates of cardioversion when used as first-line monotherapy in 5/7 (71.4\%) and $3 / 6(50 \%)$ women although this comprised only $23 \%$ 


\begin{tabular}{|c|c|c|}
\hline & Total & $\begin{array}{l}\text { Percentage of } \\
\text { cohort, } \%\end{array}$ \\
\hline Demographics & 69 & \\
\hline No transplacental therapy & 13 & 18.8 \\
\hline Delivered at diagnosis & 10 & \\
\hline Declined treatment & 1 & \\
\hline Clinical decision not to treat & 2 & \\
\hline Antenatally treated SVT & 56 & 81 \\
\hline Hydrops & 23 & 33.3 \\
\hline Nulliparous & 26 & 37.6 \\
\hline Multiparous & 41 & 59.4 \\
\hline Gestation at diagnosis, weeks+days & $29+2(26+0-33+4)$ & \\
\hline Gestation at delivery, weeks+days & $36+1(34+0-38+4)$ & \\
\hline Birth weight, $g$ & $2,940(2,668.5-3,422)$ & \\
\hline \multicolumn{3}{|l|}{ Diagnosis } \\
\hline Atrial flutter & 7 & 10 \\
\hline SVT & 62 & 89.8 \\
\hline No structural cardiac anomaly & 68 & 98.5 \\
\hline \multicolumn{3}{|l|}{ Mode of delivery $(n=58)$} \\
\hline Caesarean section & 38 & 65.5 \\
\hline Elective & 26 & 68 \\
\hline Emergency & 11 & 28.9 \\
\hline Unknown & 1 & \\
\hline Vaginal delivery & 20 & 35.1 \\
\hline \multicolumn{3}{|l|}{ Delivery indication $(n=55)$} \\
\hline Immediate delivery, no therapy & 10 & 18 \\
\hline Obstetric indication & 16 & 29 \\
\hline Maternal indication (side effects) & 4 & 7.3 \\
\hline Worsening fetal condition & 6 & 10.9 \\
\hline Rate reverted SVT at term & 19 & 34.5 \\
\hline \multicolumn{3}{|l|}{ Outcome } \\
\hline Delivered without therapy & 13 & 18.8 \\
\hline Rate reverted SVT at delivery & 46 & 66.7 \\
\hline Persistent SVT despite therapy & 7 & 10.1 \\
\hline IUFD & 3 & 4.3 \\
\hline
\end{tabular}

Data are presented as number and percentage for binary variables or median and interquartile range. SVT, supraventricular tachycardia.

of the treated cohort of 56 cases. The median latency to rate reversion was only 3 days (2-4) in the flecainide cohort and 6 days (3.5-10) in the sotalol cohort.

Of the 23 cases that had hydrops, 21 received antenatal treatment with the other 2 women delivered because of their relatively advanced gestation at diagnosis. Polytherapy was used more frequently as first-line treatment when fetal hydrops was present $(11 / 21,52 \%)$. Digoxin and flecainide in combination were used in 7 cases and were successful in correcting the FHR in 3 cases $(42.8 \%)$ at a median of 5 days (range 4-6) whilst digoxin and sotalol were used together in 4 cases. Of these, cardioversion was achieved in only 1 case. Of the remaining 10 cases of hydrops, monotherapy with either flecainide or digoxin was used with successful reversion of the SVT in 5 cases.
Overall, treatment in the context of fetal hydrops was successful in $42.9 \%(9 / 21)$ of cases.

Side effects occurred in 14 (25\%) of the 56 treated patients (Table 3), although in 6 women, these side effects were mild. The remaining 8 patients required either alternative agents or delivery due to significant side effects most commonly occurring with digoxin and flecainide polytherapy. Of women receiving digoxin and flecainide, 2 were delivered for severe nausea, 2 had therapy changed for palpitations with detectable QT prolongation on 12lead ECG, and 2 had deranged liver function which resolved with alternative agents.

There were 3 (4.3\%) fetal deaths amongst the cohort all of which were hydropic at initial presentation. Of these, although rate reversion was achieved in 2 fetuses 


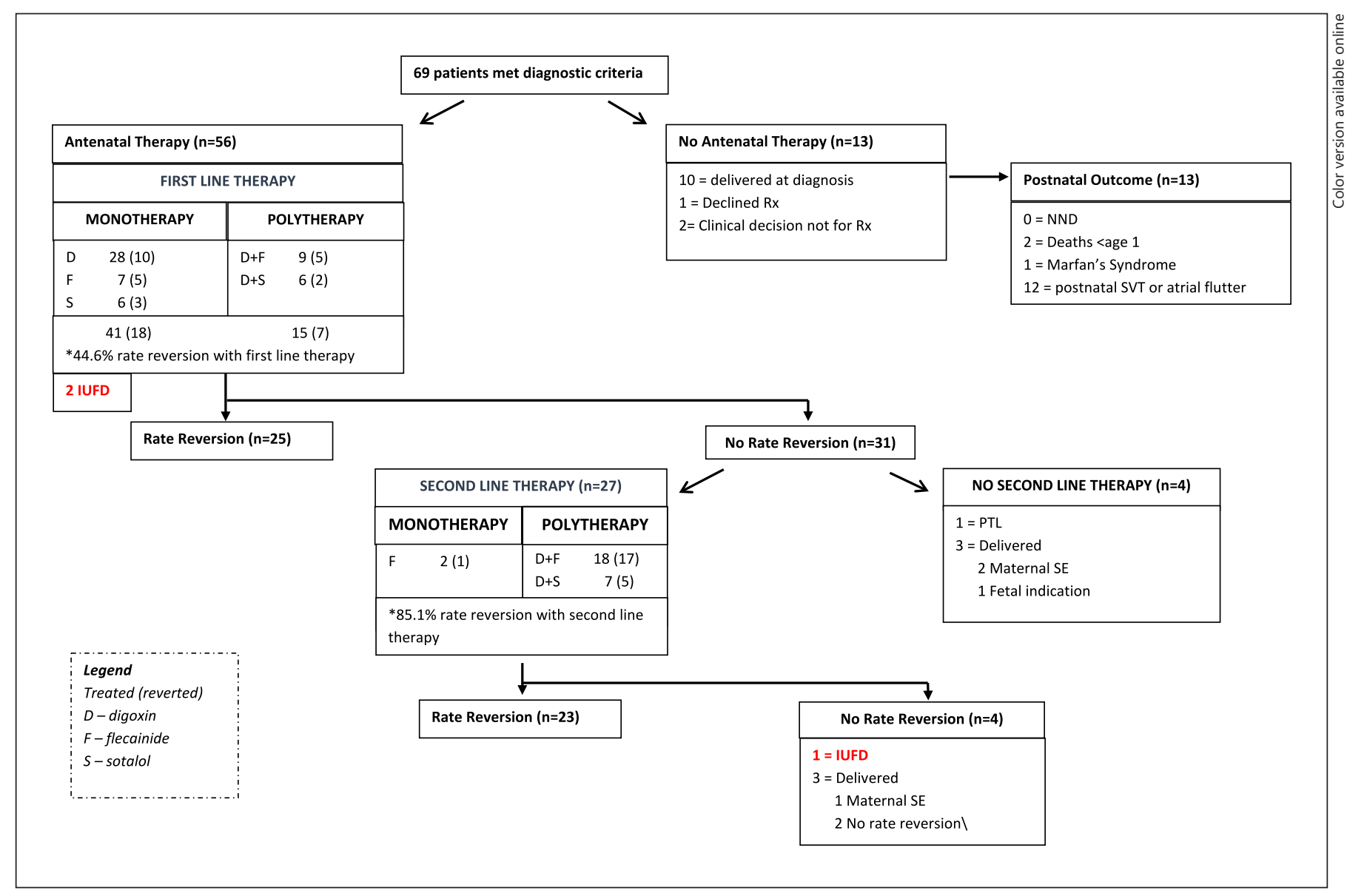

Fig. 1. Study criteria and outcomes.

using digoxin and flecainide, respectively, hydrops did not resolve and fetal demise occurred at 10 days and 35 days, respectively, following treatment. In the third case, despite 3 different agents including amiodarone, the SVT remained refractory, and fetal death was diagnosed 10 days following initial therapy. A further 6 fetuses (8.7\%) were delivered on the basis of worsening hydrops or abnormal Doppler parameters.

The median gestation at delivery was $36+1$ weeks $(34+0-38+5)$. Of the 58 women in the study cohort for whom mode of delivery details were available, 38 (65.5\%) were delivered by caesarean section. However, the indication for recommending delivery was only documented in 55 women. Of these 55 women, 10 (18\%) were delivered for maternal side effects or worsening fetal condition, and a further 10 (18\%) delivered immediately without therapy. In $10(18 \%)$ women, preterm labor or preterm prelabor rupture of membranes precipitated delivery.

\section{Discussion}

In this large, single-center study, we demonstrate high success rates for treatment of fetal SVT when digoxin and flecainide were used in combination. Overall, this dualagent regimen achieved rate reversion in 22 of 27 (81.5\%) cases. Our results are comparable to other published series which report success rates of 59-90\% using digoxin, flecainide, or sotalol in combination [9-13]. Our results also indicate that in almost 1 in 3 cases without hydrops and 1 in 2 cases with hydrops, initial monotherapy failed thus requiring progression to second-line polytherapy.

Digoxin was the first-line agent in the majority of cases and achieved rate reversion in $35.7 \%$ in line with other published series $[3,10,14]$. Digoxin was the preferred initial treatment because of its safety and side effect profile and relatively rapid response time $[10,11]$. However, we note that studies reporting high treatment efficacy of digoxin used much higher daily doses of up to $750 \mu \mathrm{g} /$ day [11], as 


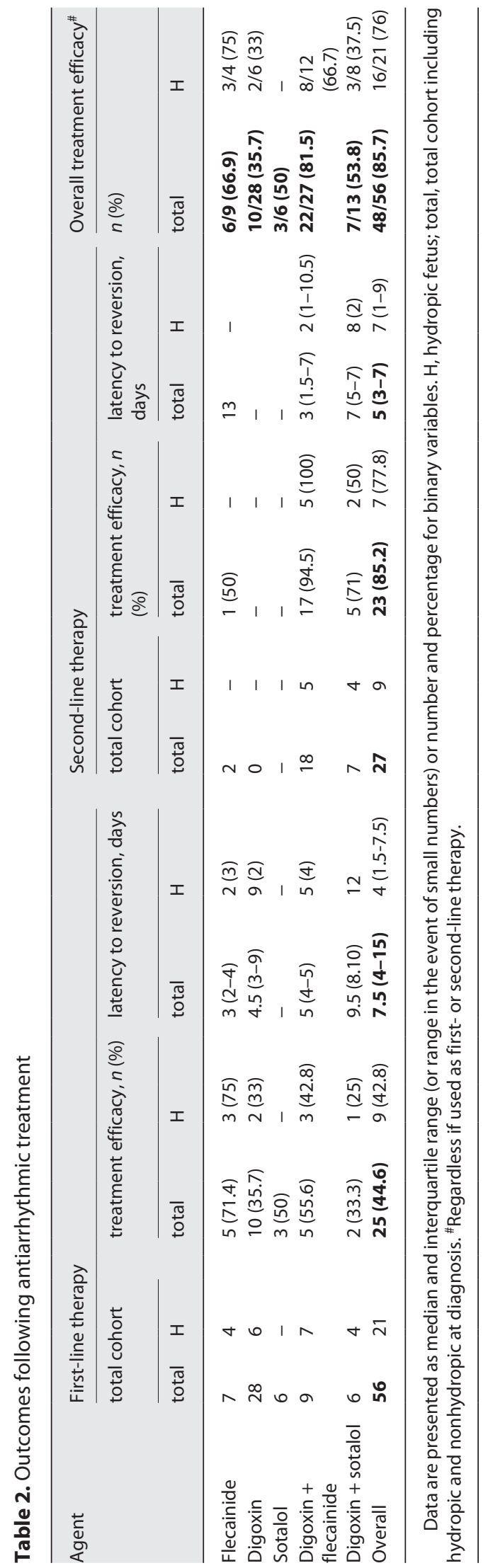

opposed to only $500 \mu \mathrm{g} /$ day in our cohort. When hydrops was present, treatment efficacy in achieving rate reversion was higher (5/7 cases, $71.4 \%$ ) when digoxin and flecainide were used in combination. Our results are consistent with other published outcomes that range from 59 to $78 \%$ using this approach [14-18]. However, dosing regimens in the literature vary significantly $[2,7,9,10,15]$, and interval to fetal rate reversion from diagnosis may be longer with polytherapy. This likely reflects current practice in utilizing polytherapy mainly after failed first-line monotherapy [17].

Our results demonstrate that rate reversion occurred at a median of 7.5 days (4-15) of starting maternal treatment. Second-line treatment was trialled by 7 days of failure of rate reversion in all by 8 of 56 fetuses. The indication for addition of a second agent was lack of fetal response in $90 \%$ of cases, with worsening fetal condition observed in only 2 fetuses over this initial treatment time period.

Overall, maternal side effects were relatively uncommon in our cohort, reported in only $25 \%$ of women. Serious side effects warranting dose adjustment or delivery were encountered in $14.3 \%$ of patients. Although women on flecainide monotherapy did not report any side effects, this was not the case when it was used in combination with other agents. In 6 out of 8 cases of serious maternal side effects, flecainide was implicated. Significant maternal side effects were seen when the dose of flecainide was $>300 \mu \mathrm{g} /$ day. Likewise, sotalol at a dose of $>160 \mathrm{mg} /$ day was associated with $50 \%$ of side effects attributable to this drug. In general, maternal serum levels of digoxin and flecainide aid more in monitoring for maternal toxicity rather than as a guide to therapeutic fetal levels as these have yet to be quantified [19]. The higher maternal side effect profile of flecainide is often cited as a reason to use alternative first-line agents $[14,15,18,20]$.

In this series, cardioversion was only possible in $42.9 \%$ (9/21 cases) of hydropic fetuses following treatment. Fetal hydrops is associated with increased fetal volume of distribution - this is believed to be responsible for the lack of efficacy when digoxin is used in this setting $[15,19,21]$ $[10,15,22]$. Consistent with other studies, we were unable to ascertain if polytherapy with digoxin and another agent was superior to digoxin monotherapy alone [23]. Historically, mortality rates amongst hydropic fetuses on treatment have been reported to be as high as $27 \%$ [2], though more recent case series report more conservative rates around 5\% [9]. We report an overall mortality rate of $4.3 \%(3 / 69)$, though this was $13 \%(3 / 23)$ amongst the cohort with hydrops at diagnosis.

The low rate of subclassification of SVT amongst our cohort makes it difficult to draw conclusions regarding 
Table 3. Maternal side effect profile

\begin{tabular}{|c|c|c|c|c|}
\hline Digoxin & 1 & 0 & & $\mathrm{D} 625 \mu \mathrm{g} \mathrm{BD}$ \\
\hline Flecainide & 0 & 0 & & \\
\hline Amiodarone & 0 & 1 & (1) Palpitations and chest pain & \\
\hline Digoxin + flecainide & 2 & 6 & $\begin{array}{l}\text { (2) Nausea (necessitating } \\
\text { delivery) } \\
\text { (2) QT prolongation } \\
\text { (2) Deranged LFTs }\end{array}$ & $\begin{array}{l}\text { (D } 250 \mu \mathrm{g} \mathrm{BD}, \mathrm{F} 100 \mathrm{mg} \text { BD) (D } 250 \mu \mathrm{g} \mathrm{BD}, \mathrm{F} 100 \mathrm{mg} \\
\text { TDS) } \\
\text { (D } 250 \mu \mathrm{g} \text { BD, F } 100 \mathrm{mg} \text { BD) (D } 250 \mu \mathrm{g} \mathrm{BD}, \mathrm{F} 100 \mathrm{mg} \\
\text { TDS) } \\
\text { (D } 250 \mu \mathrm{g} \text { TDS, F } 100 \mathrm{mg} \text { TDS) (D } 250 \mu \mathrm{g} \text { OD, F ?) }\end{array}$ \\
\hline
\end{tabular}

Three patients delivered due to intolerable side effects. All >34+0 at time of delivery. $D$, digoxin; F, flecainide; S, sotalol; OD, once daily dosing; BD, twice daily dosing; TDS, 3 times daily dosing.

subtype response to therapy. We postulate that although the underlying mechanism of SVT may influence fetal response [24], this impact may have been underestimated in the past, and so subclassification diagnosis was not often documented. Sotalol as first-line treatment was our preference for long VA SVT. This approach is supported by data showing that both digoxin and flecainide monotherapy for long VA SVT is associated with a longer delay to reversion and poorer response [23, 24]. Accurate identification and subclassification of SVT through characterization of the VA interval at fetal echocardiography may highlight subgroups such as atrial flutter, which are more likely to respond to polytherapy as first-line treatment.

Cases refractory to treatment represent a therapeutic challenge. Only 3 (4.3\%) cases in our series demonstrated persistent SVT. Although both oral and intravenous amiodarone is an effective agent for the treatment of fetal SVT, its side effect profile and the need for careful maternal monitoring preclude its use as first-line therapy, and it is normally reserved for refractory cases [25]. However, in 2 of our 3 refractory cases, amiodarone also failed to achieve rate reversion. Although it was not used in our cohort, intra-amniotic digoxin has been used prenatally, with rapid resolution of fetal SVT [26]. This may be an alternative option at very preterm gestations when delivery is not an option.

Once rate and rhythm control was achieved, vaginal birth was offered. There were no cases of recurrent fetal
SVT in labor. Whether therapeutic rate control with transplacental therapy impairs accurate detection of fetal hypoxia in labor manifested by cardiotocographic changes is unknown. In our series, of the 11 emergency caesarean sections, only 4 were performed in labor. Unfortunately, markers of fetal hypoxia such as cord artery $\mathrm{pH}$ were not available for all cases. Limitations of our study include its retrospective nature, treatment preferences by individual cardiologists, and lack of some outcome data.

\section{Conclusion}

Robust protocols to guide treatment of fetal SVT are lacking. Although several antiarrhythmic options and treatment protocols exist in the literature, refractory cases and nonresolution of hydrops despite rate reversion remain therapeutic challenges. Key to optimizing perinatal outcomes is rapid rate reversion using the lowest maternal dose, thus minimizing maternal side effects and maximizing compliance. Recognition of antenatal factors predisposing to treatment failure is important in deciding the choice of initial treatment. We await the outcomes of the Fetal Atrial Flutter and Supraventricular Tachycardia Therapy Randomised Clinical Trial (FAST RCT) (Clinical Trials Identifier NCT02624765) which will hopefully provide guidance as to the optimum management for these difficult cases. 


\section{Acknowledgments}

The authors wish to acknowledge the contributions of Dr. Mugurel Nicolae to the editing and collation of this manuscript and to the staff of the Maternal Fetal Medicine Department at the Mater Mother's Hospital to data collection and patient care.

\section{Statement of Ethics}

Ethics exemption and Governance approvals (HREC/14/ MHS/37) were granted by the Mater Human Research Ethics Committee and Governance Office, respectively. Specifically, the ethics exemption includes waiver of patient consent for audit purposes.

\section{Conflict of Interest Statement}

The authors have no conflicts of interest to declare.

\section{Funding Sources}

This work received no internal or external funding.

\section{Author Contributions}

All named authors contributed to the conception of the research project and to the drafting and revision of the final manuscript.

\section{Data Availability Statement}

All data analyzed in this study are included in this article. Further enquiries can be directed to the corresponding author.

\section{References}

1 Jaeggi E, Öhman A. Fetal and neonatal arrhythmias. Clin Perinatol. 2016;43:99-112.

2 Simpson LL, Marx GR, D’Alton ME. Supraventricular tachycardia in the fetus: conservative management in the absence of haemodynamic compromise. J Ultrasound Med. 2009; 16:459-64.

3 Karmegeraj B, Namdeo S, Sudhakar A, Krishnan V, Kunjukutty R, Vaidyanathan B. Clinical presentation, management, and postnatal outcomes of fetal tachyarrhythmias: a 10-year single-center experience. Ann Pediatr Cardiol. 2018;11(1):34-9.

4 Maeno Y, Hirose A, Kanbe A, Hori D. Fetal arrhythmia: prenatal diagnosis and perinatal management. J Obstet Gynaecol Res. 2009; 35(4):623-9.

5 Strizek B, Berg C, Gottschalk I, Herberg H, Geipel A, Gembruch U. High dose flecainide is the most effective treatment of fetal supraventricular tachycardia. Heart Rhythm. 2016; 13:1283-8.

6 Naheed ZJ, Strasburger JF, Deal BJ, Benson DW, Gidding SS. Fetal tachycardia: mechanisms and predictors of hydrops fetalis. J Am Coll Cardiol. 1996;27:1736-40.

7 Cuneo BF. Treatment of fetal tachycardia. Heart Rhythm. 2008;5(8):1216-8.

8 Carvalho JS. Fetal dysrhythmias. Best Pract Res Clin Obstet Gynaecol. 2019;58:28-41.

9 Uzun O, Babaoglu K, Sinha A, Massias S, Beattie B. Rapid control of foetal supraventricular tachycardia with digoxin and flecainide combination treatment. Cardiol Young. 2012; 22(4):372-80

10 Alsaied T, Baskar S, Fares M, Alahdab F, Czosek RJ, Murad MH, et al. First-line antiarrhythmic transplacental treatment for fetal tachyarrhythmia: a systematic review and meta-analysis. J Am Heart Assoc. 2017;6(12):e007164.
11 Miyoshi T, Maeno Y, Hamasaki T, Inamura N, Yasukochi S, Kawataki M, et al. Antenatal therapy for fetal supraventricular tachyarrhythmias: multicenter trial. J Am Coll Cardiol. 2019;74(7):874-85.

12 Oudijk MA, Michon MM, Kleinman CS, Kapusta L, Stoutenbeek P, Visser GH, et al. Sotalol in the treatment of fetal dysrhythmias. Circulation. 2000;101:2721-6.

13 O'Leary ET, Alexander ME, Bezzerides VJ, Drogosz M, Economy KE, Friedman KG, et al. Low mortality in fetal supraventricular tachycardia: outcomes in a 30-ear single: institution experience. J Cardiovasc Electrophysiol. 2020;31(5):1105-13.

14 Cuneo BF, Benson DW. Use of maternal flecainide concentration in management of fetal supraventricular tachycardia: a step in the right direction. Heart Rhythm. 2014;11(11):2054-5.

15 Strizek B, Berg C, Gottschalk I, Herberg U, Geipel A, Gembruch U. High-dose flecainide is the most effective treatment of fetal supraventricular tachycardia. Heart Rhythm. 2016; 13(6):1283-8

16 Jaeggi ET, Carvalho JS, De Groot E, Api O, Clur SA, Manlhiot C, et al. Comparison of efficacy and safety of first-line transplacental treatment of fetal supraventricular tachycardia (SVT) and atrial flutter (AF) with sotalol, flecainide and digoxin. Circulation. 2010;122(21):2.

17 Jaeggi ET, Carvalho JS, De Groot E, Api O, Clur SA, Rammeloo L, et al. Comparison of transplacental treatment of fetal supraventricular tachyarrhythmias with digoxin, flecainide, and sotalol: results of a nonrandomized multicenter study. Circulation. 2011;124(16):1747-54.

18 Ekiz A, Kaya B, Bornaun H, Acar DK, Avci ME, Bestel A, et al. Flecainide as first-line treatment for fetal supraventricular tachycardia. J Matern Fetal Neonatal Med. 2018;31(4):407-12.
19 Martin-Suarez A, Sanchez-Hernandez JG, Medina-Barajas F, Pérez-Blanco JS, Lanao JM, Garcia-Cuenllas Alvarez L, et al. Pharmacokinetics and dosing requirements of digoxin in pregnant women treated for fetal supraventricular tachycardia. Expert Rev Clin Pharmacol. 2017;10(8):911-7.

20 Jaeggi E, Carvalho JS, De Groot E, Api O, Clur $\mathrm{S}$, Manlhiot C, et al. Comparison of efficacy and safety of first-line transplacental treatment of fetal supraventricular tachycardia and atrial flutter with sotalol, flecainide and digoxin. Circulation. 2010;122(21):2.

21 Saad AF, Monsivais L, Pecheco LD. Digoxin therapy of fetal supraventricular tachycardia: are digoxin serum levels reliable? AJP Rep. 2016;06(3):272-6.

22 Zoeller BB. Treatment of fetal supraventricular tachycardia. Curr Treat Options Cardiovasc Med. 2017;19(1):7.

23 Sridharan S, Sullivan I, Tomek V, Wolfenden J, Škovránek J, Yates R, et al. Flecainide versus digoxin for fetal supraventricular tachycardia: comparison of two drug treatment protocols. Heart Rhythm. 2016;13(9):1913-9.

24 Strasburger JF. Predictability in fetal supraventricular tachycardia management. J Am Coll Cardiol. 2019;74(7):886-8.

25 Strasburger JF, Cuneo BF, Michon MM, Gotteiner NL, Deal BJ, McGregor SN, et al. Amiodarone therapy for drug-refractory fetal tachycardia. Circulation. 2004;109:375-9.

26 López Ramón y Cajal C, Couceiro Naveira E, Piñón Mosquera A, Gómez Mosquera A. Treatment of fetal supraventricular tachycardia by intra-amniotic administration of digoxin. Ultrasound Obstet Gynecol. 2019;54(5):698-9.

Broom/Thomas/Petersen/Gooi/Ward/ Gardener/Kostner/Lee-Tannock/Kumar 